\title{
El póster académico como instrumento innovador en la enseñanza de inglés para fines específicos Innovation in English for Specific Purposes through Academic Posters
}

\author{
Beatriz Martín Marchante, Ana María Gimeno Sanz \\ beamarm2@upvnet.upv.es, agimeno@upvnet.upv.es \\ Universitat Politècnica de València, \\ Valencia, España
}

\begin{abstract}
Resumen- En este trabajo se describe la implementación del póster académico como instrumento innovador y motor de la motivación en la enseñanza de inglés para fines específicos en el grado de Ingeniería Aeroespacial en la Universitat Politècnica de València. La experiencia forma parte de un proyecto de innovación y mejora educativa que lleva por título "El póster académico y las TIC: herramientas para el desarrollo de competencias transversales y el aprendizaje de contenidos curriculares y lenguas para fines específicos", que se puso en marcha en la Escuela Técnica Superior de Ingeniería del Diseño en el curso 2020-21. Las autoras abordan los resultados obtenidos a través de una encuesta inicial y una final en las que se exploraban las expectativas al inicio del proyecto y el grado de satisfacción tras su realización, respectivamente. Por otra parte, se presentarán los datos obtenidos respecto a la alineación del proyecto en relación con la mejora de una selección de las competencias transversales que se fomentan en las asignaturas impartidas en la Universitat Politècnica de València. Por último, se dará cuenta del grado de evolución del aprendizaje respecto a la lengua de especialidad cursada por los estudiantes.
\end{abstract}

Palabras clave: Póster académico, competencias transversales, inglés para fines específicos

Abstract- This study focuses on implementing the academic poster as an innovative and motivating instrument in teaching English for Specific Purposes within the Aerospace Engineering undergraduate degree at Universitat Politècnica de València. It is part of a two-year teaching innovation project funded by the University: "Academic posters and ICT: tools to develop transversal skills, subject matter content and languages for specific purposes", carried out at the School of Design Engineering during the 2020-21 academic year. The results are drawn from pre- and post-project questionnaires exploring learner expectations and overall satisfaction upon completion. The authors also discuss project alignment with a number of transversal competences encouraged by the University through its courses. To conclude, the authors refer to the learners' language intake upon completion of the project.

Keywords: Academic poster, transversal competences, English for Specific Purposes

\section{INTRODUCCIÓN}

El alumnado de la Universitat Politècnica de València (UPV) debe desarrollar una serie de competencias transversales y específicas a través de distintas tareas y actividades que se enmarcan en una metodología activa. Dentro de este marco de trabajo se espera que nuestros/as estudiantes trabajen colaborativamente en equipo y sean capaces de construir conocimiento a través de proyectos que, una vez finalizados, sean presentados oralmente. Normalmente estas presentaciones orales se realizan en formato PowerPoint en las asignaturas de lengua, mientras que el póster, como señala Hyland (2000), parece valorarse y usarse más en el campo de las ciencias. Teniendo en cuenta que la mayoría de las asignaturas de lengua que se imparten en la Escuela Técnica Superior de Ingeniería del Diseño, centro en el que se ha puesto en marcha este proyecto, están concebidas y diseñadas para los usos específicos que determina cada titulación (Ingeniería Eléctrica, Electrónica, del Diseño Industrial y Desarrollo de productos, Mecánica y Aeroespacial), contemplamos la posibilidad de introducir el género póster como una alternativa viable, en tanto que los temas que habitualmente se desarrollan en los proyectos de clase son de naturaleza científico-técnica. Algunos profesores venían observando que las presentaciones orales con PowerPoint conllevaban algunas desventajas que cabía reconsiderar. Nos referimos al hecho de que las condiciones espaciotemporales en las que tienen lugar las sesiones de presentación con este tipo de soporte visual (o similar) conlleva ciertas barreras psicológicas, tales como las producidas por la diferencia de nivel espacial: superior (tarima) e inferior (asientos para el público), o por perspectivas visuales confrontadas. Gran parte de nuestro alumnado afirma que esta situación resulta estresante y para algunos también intimidante, efectos que no son de extrañar si tenemos en cuenta que a muchos/as de ellos/as les supone un gran esfuerzo hablar en público, o bien no están habituados/as a hacerlo.

Por otra parte, el profesorado ha comprobado que muy a menudo más que un verdadero trabajo colaborativo, lo que se produce es la suma de diapositivas creadas de manera individual a pesar de los mecanismos y estrategias que se llevan a cabo para intentar evitarlo. Esta situación nos ha llevado a pensar en el póster académico como un recurso alternativo a la presentación con PowerPoint o similar, ya que numerosos autores (Rosell, Trenado y Simó, 2011; García Folgado, 2017; Navarro y Félix, 2017 y García Manso, 2019), sostienen que se trata de una herramienta pedagógica eficiente.

Otros autores (Moore, Augspurger, King y Proffitt, 2001; Shelledy, 2004; Halligan, 2008), defienden que la presentación de un trabajo colaborativo en forma de póster académico puede 
resultar estimulante, puesto que sitúa al comunicador y a la audiencia en un mismo nivel, facilita el intercambio de ideas y la comparación de resultados. Como indica D'Angelo (2010), el póster facilita el debate informal y ofrece un foro más cercano para el intercambio que el que puede ofrecer otro tipo de formatos de presentación, ya que, en una situación ideal, un póster bien construido se explica por sí mismo y libera al presentador de responder preguntas obvias, de tal manera que este/esta pueda completar y discutir aspectos de interés más concretos. También Baird (1991), citado en D'Angelo (2010), argumenta su uso como alternativa a las tareas tradicionales de escritura y piensa que las sesiones de pósteres son adecuadas para todo tipo de clases, promueven el aprendizaje colaborativo, fomentan la creatividad y el pensamiento independiente, y desarrollan habilidades de investigación y comunicación.

Por otra parte, si para la realización de un póster académico el alumnado utiliza una serie determinada de aplicaciones tecnológicas (por ejemplo, para dispositivos móviles) éste puede ver aumentada su motivación $\mathrm{y}$, consecuentemente, mejorar su rendimiento académico, como defienden algunos autores (Schoepp y Erogul, 2001).

El objetivo del presente estudio es analizar el grado de satisfacción de los participantes tras la elaboración de un póster académico y su posterior utilización como soporte visual para las presentaciones orales de sus proyectos. Así mismo, se pretende medir el grado de adquisición de competencias transversales y mejora del aprendizaje de la lengua de especialidad según la percepción de los sujetos de la investigación. Para dar cuenta de cómo se han abordado los objetivos en el presente artículo se describe, en primer lugar, el contexto; seguidamente se explica la metodología utilizada y se presentan los resultados obtenidos. Finalmente, se exponen las conclusiones a las que se ha llegado tras el análisis de los datos obtenidos.

\section{CONTEXTO}

A lo largo del segundo semestre del curso 2020-21, se puso en marcha en la asignatura de Inglés Técnico para la Ingeniería Aeroespacial el proyecto del póster académico. Esta asignatura optativa de 1 er curso tiene un peso de 6 créditos, de los cuales 3 son de teoría y 3 de práctica. El proyecto se integró dentro de los créditos de teoría y se relacionó con uno de los temas que se tratan en el libro de texto que se utiliza (Ready for Take-Off: The Language of Aeronautics de C. Douglas Billet, publicado en 2001 por Media Training Corporation, Cannes). El peso en la evaluación final de la asignatura fue de un $20 \%$.

El grupo estaba integrado por 28 estudiantes, de entre 18 y 20 años, distribuidos en siete equipos de trabajo. Su nivel de inglés oscilaba entre el nivel B1 y C1 del Marco común europeo de referencia para las lenguas.

\section{DESCRIPCIÓN}

La experiencia se distribuyó a lo largo de 10 semanas dentro del $2^{\circ}$ semestre de 1 er curso. Tras la presentación del proyecto, se les facilitó a través de la intranet el calendario a seguir y las actividades a realizar. Durante las primeras cuatro sesiones se les fue explicando las pautas a seguir, así como las herramientas a utilizar. Asimismo, se les instruyó sobre técnicas de creación de pósteres académicos. A medida que fueron disminuyendo las directrices sobre el proyecto, fue aumentando el trabajo autónomo de los grupos de trabajo hasta culminar con la impresión y exposición pública de los pósteres realizados.

Las etapas que conformaron el proyecto de mejora docente para los estudiantes fueron las siguientes:

a) Investigación sobre el tema y recopilación de material gráfico.

b) Redacción del artículo a partir del cual realizar el póster.

c) Diseño y realización del póster académico.

d) Impresión y exposición del póster.

e) Presentaciones orales de cada equipo en el aula.

La recogida de datos se realizó a través de un cuestionario inicial para recabar la experiencia previa del alumnado en la realización de un póster académico y para conocer sus expectativas ante el proyecto, y a través de un cuestionario final para conocer la opinión del alumnado y su grado de satisfacción tras su realización. Del total de 28 estudiantes, 22 de ellos realizó el cuestionario inicial y 24 , el final.

Asimismo, se recogió información sobre la percepción de los participantes respecto a la alineación del proyecto en relación con la mejora de una selección de las competencias transversales que se fomentan en la UPV, y cuyo grado de adquisición se valora en las asignaturas.

Todos los cuestionarios se administraron a través de Google Forms y su cumplimentación fue de carácter obligatorio y sin ser anónimo. Todas las preguntas se contestaron sobre una escala de 7 puntos, siendo 1 el valor más bajo (totalmente en desacuerdo) y 7 , el valor más alto (totalmente de acuerdo). El cuestionario inicial constó de 25 preguntas. Con las primeras 8 se recogieron datos demográficos y datos sobre los hábitos de consumo de internet. Las siguientes 8 se centraban en la experiencia previa del póster académico como herramienta didáctica y las expectativas sobre dicha actividad en clase. Una pregunta tenía como objetivo conocer la motivación del estudiantado hacia la asignatura y 4 de ellas tenían como objetivo recabar información sobre la disposición del estudiantado a trabajar en equipo y de forma colaborativa. Por último, una pregunta indagaba sobre la relación de la realización del póster académico con el uso de las tecnologías de la información y de la comunicación, otra, para ver si los estudiantes percibían la actividad como un estimulante para formar parte de una comunidad de práctica y, por último, una pregunta para conocer si pensaban que la experiencia favorecería la relación social entre compañeros y compañeras de clase.

Por otra parte, el cuestionario final constó de 26 ítems, que indagaron sobre las siguientes cuestiones. Además de las mismas 8 preguntas demográficas, hubo una pregunta sobre el grado de seguridad en la signatura de inglés. Además, se les preguntó cuántas horas estimaban que habían empleado en el proyecto y su grado de satisfacción respecto a trabajar en equipo de forma colaborativa. Otras 7 preguntas cuestionaban la percepción de los estudiantes respecto a la experiencia del proyecto del póster académico y sobre el grado de ansiedad que la presentación en público pudo suponerles. Por últimos, los siguientes 7 ítems indagaban sobre los aspectos que, en opinión de los participantes, habían mejorado tanto en términos de la adquisición de la lengua extranjera, como respecto a una serie de competencias transversales. 
La evaluación de la actividad se llevó a cabo por las docentes a través de una rúbrica realizada ad hoc. Además, tras la exposición pública de los pósteres, los estudiantes realizaron preguntas e hicieron comentarios a propósitos del tema expuesto simulando el contexto habitual de presentación de un póster académico.

\section{Resultados}

Del cuestionario inicial pudimos saber que un $41 \%(\mathrm{~N}=9)$ de los estudiantes sabían lo que era un poster académico y, en igual medida, que habían realizado uno con anterioridad, mientras que un $59 \%(\mathrm{~N}=13)$ ni sabía qué era ni tenía experiencia previa alguna.

Los estudiantes partían de una actitud positiva ante la experiencia ya que, en una escala de 1 a 7 (siendo 1 "totalmente en desacuerdo" y 7 "totalmente de acuerdo"), ninguno indicó la mitad negativa de la escala. La suma de opinión favorable (valores 5 a 7) ascendió a un $82 \%(\mathrm{~N}=22)$, como se puede ver en la figura 1.

La creación de un póster académico como tarea de trabajo en equipo para clase puede resultar divertido

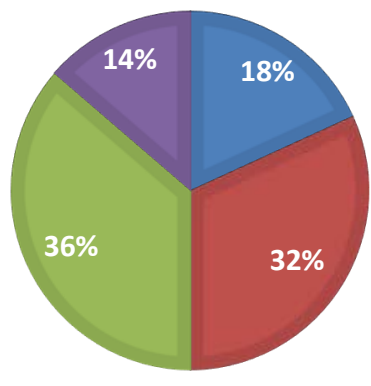

$\square \square \square \square 6 \square 7$

Figura 1. Pregunta 12 del cuestionario inicial.

Las expectativas que tenían nuestros estudiantes respecto a la mejora de la lengua inglesa fueron diversas ya que, como se puede apreciar en la figura 2, todas las destrezas y elementos lingüísticos estaban representadas en sus expectativas de aprendizaje. Un $24 \%$ opinó que la experiencia le ayudaría a aprender más gramática; un $23 \%$, que adquiriría más vocabulario; un $20 \%$, que le ayudaría a mejorar su producción escrita; un $17 \%$, su producción oral; un $12 \%$, la comprensión oral y, en último lugar, con un 4\%, la comprensión oral. Estos datos nos indican que los estudiantes relacionan la creación de un póster académico y su exposición ante un público con la adquisición de gramática y vocabulario que, con toda probabilidad, asocian como base fundamental para la mejora de las cuatro destrezas. Estos datos nos indican que sus expectativas de mejora están relacionadas en mayor medida con las destrezas productivas.

Por otra parte, no hubo correlación entre el nivel lingüístico declarado y las expectativas de mejora de la lengua, ni tampoco hubo correlación entre la experiencia previa en la creación de un poster académico y las expectativas de mejora de algún aspecto concreto de la lengua inglesa.
La creación de un póster académico en inglés me ayudará a:

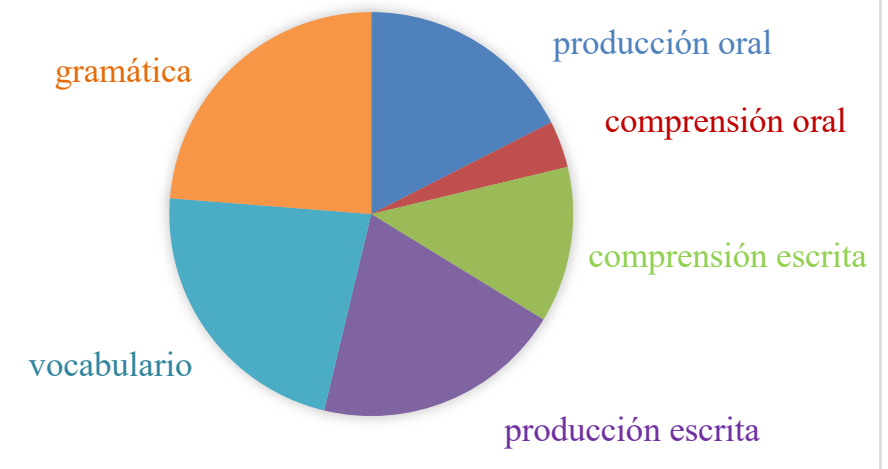

Figura 2. Pregunta 15 del cuestionario inicial.

Paralelamente, en el cuestionario final también cobraron más peso las destrezas productivas, superándose las expectativas de aprendizaje de vocabulario y disminuyéndose las de gramática (Fig. 3). En las destrezas pasivas no hubo cambios destacables.

Crear un póster académico en inglés me ha ayudado a mejorar:



Figura 3. Pregunta 17 del cuestionario final.

En lo que se refiere a la percepción sobre la mejora de destrezas no lingüísticas del proyecto, cabe señalar los resultados de la figura 4 .

\section{En general, la creación de un póster académico me ayudará a:}

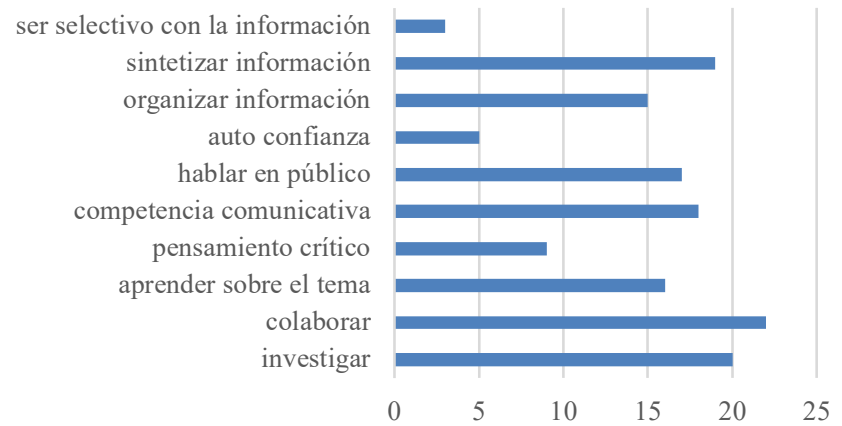

Figura 4. Pregunta 16 del cuestionario inicial.

Como se puede apreciar (Fig. 4), por orden de importancia, pensaban que les ayudaría a colaborar con otros estudiantes, a investigar sobre el tema propuesto, a sintetizar información, a desarrollar su competencia comunicativa, a hablar en público, a aprender sobre el tema propuesto, a organizar información y, en menor medida, a fomentar el pensamiento crítico, generar auto 
confianza y a ser selectivo con la información que se maneja y entresacar lo más importante.

Comparado con los resultados después del proyecto (Fig. 5), el análisis revela que, en general, todos los valores han aumentado, lo cual indica que la realización de un póster académico dentro de la asignatura de inglés para fines específicos ha ayudado a los estudiantes a adquirir o mejorar las destrezas no lingüísticas que se perseguían en mayor medida de lo que inicialmente imaginaban. Este hecho se considera como un logro.

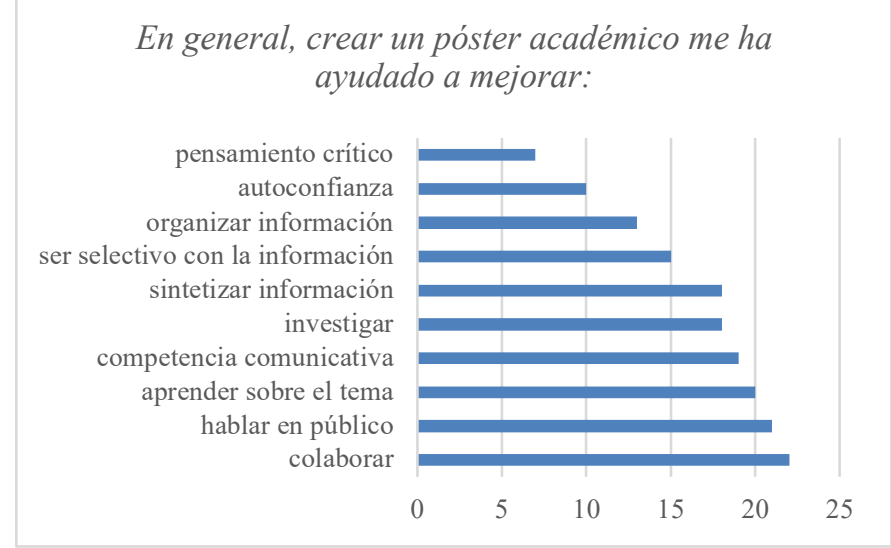

Figura 5. Pregunta 18 del cuestionario final.

Fueron una mayoría (95\%) quienes opinaron que les gustaba el trabajo en grupo y colaborar con otros estudiantes en clase (Fig. 6). Sólo 1 (5\%) indicó estar "más bien en desacuerdo" (valor 3 de la escala).

Por otro lado, cuando se les pregunto si preferían trabajar en grupo o de forma individual (pregunta 22), hubo un $27 \%$ que señaló la franja negativa de la escala, indicando así, su tendencia a la disconformidad al trabajo en grupo.

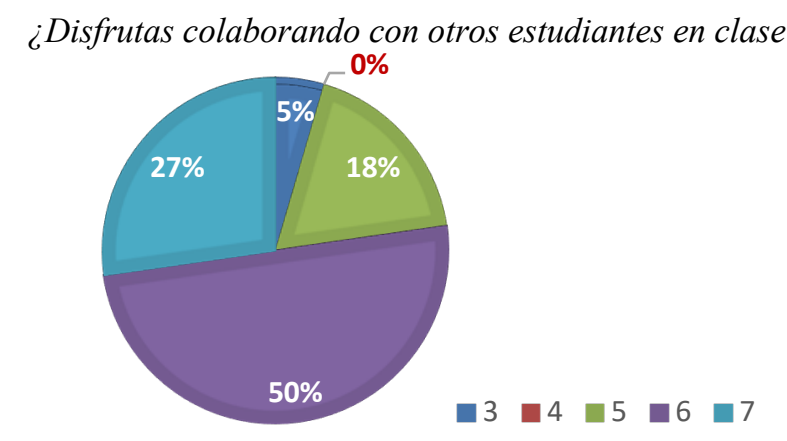

Figura 6. Pregunta 19 del cuestionario inicial.

Un $18 \%(\mathrm{~N}=4)$ de los estudiantes manifestaron cierto grado de ansiedad a la hora de enfrentarse a este elemento novedoso dentro del aula de inglés, aunque la media general fue de 3 (en una escala de 7 puntos), equivalente a "más bien en desacuerdo". Tras la realización del proyecto, todos menos 1 alumno $(4,1 \%)$, opinaron haber superado la ansiedad generada al comienzo del proyecto.

La mayoría de los estudiantes reconoció haber dedicado entre 3 y 5 horas de trabajo fuera del aula al proyecto ya que parte de la clase presencial se reservó para las diversas fases de la actividad.
Dado que uno de los problemas más comunes cuando se les pide a los estudiantes que realicen un trabajo en grupo es la descompensación del tiempo y del esfuerzo que cada miembro del grupo dedica para sacar adelante el trabajo, en el cuestionario final se les preguntó el grado de satisfacción respecto a trabajo colaborativo. Exceptuando un 16,6\% $(\mathrm{N}=4)$, que indicó "bastante satisfecho", el restante $83,4 \%(\mathrm{~N}=20)$ manifestó estar "totalmente satisfecho". Esto se correlaciona con el hecho de que en el cuestionario inicial todos excepto 4 pensaban que iban a disfrutar con el proyecto realizándolo de forma colaborativa (véase Fig. 1). También se alinea este dato con el hecho de que un 5\% (véase Fig. 6) indicó, de partida, preferir no trabajar en equipo sino de forma individual.

Como una parte importante de la experiencia se basó en la presentación oral del póster en clase ante los compañeros, nos interesó saber si, al acabar la experiencia, preferían el modo "tradicional" de realizar las presentaciones orales en el aula con PowerPoint o si, por el contrario, optaban por esta nueva fórmula. Un $70 \%(\mathrm{~N}=17)$ dijo preferir la modalidad del póster académico. Una de las explicaciones aportadas es esclarecedora al respecto: "Because I think it is a much more dynamic, entertaining and fun way to carry out research and explain our results later" (Traducción de las autoras: "Porque creo que es una forma mucho más entretenida y divertida de realizar una investigación y explicar los resultados después"). No hemos de despreciar, sin embargo, que un $30 \%$ optó por el modo al que están más acostumbrados. Por ejemplo, una de las razones alegadas fue: "I find the PowerPoint a faster way to prepare presentations, because although the poster can be more original and dynamic, it requires a higher amount of time to prepare it."

Como uno de los objetivos del proyecto de mejora docente también iba dirigido a que los estudiantes se implicaran activamente en la actividad ayudándose, colaborando para lograr unos objetivos comunes, una de las preguntas en ambos cuestionarios indagó sobre el grado en el que pensaban que el proyecto podía contribuir a que sintieran que formaban parte de una comunidad de aprendizaje y si, al final, había sido así. En la figura 7 podemos comprobar que la gran mayoría de ellos marcó la parte favorable de la escala de 7 puntos. En total, un $75 \%(\mathrm{~N}=18)$ y sólo un estudiante $(4 \%)$ indicó el valor 3 equivalente a "más bien en desacuerdo", lo cual es comprensible cuando descubrimos que ese mismo estudiante indicó, asimismo, que sólo había trabajado entre 1 y 2 horas en el proyecto fuera de las horas de clase.

\section{Crear un póster académico en colaboración con los compañeros me ha hecho sentir parte de una comunidad de aprendizaje}

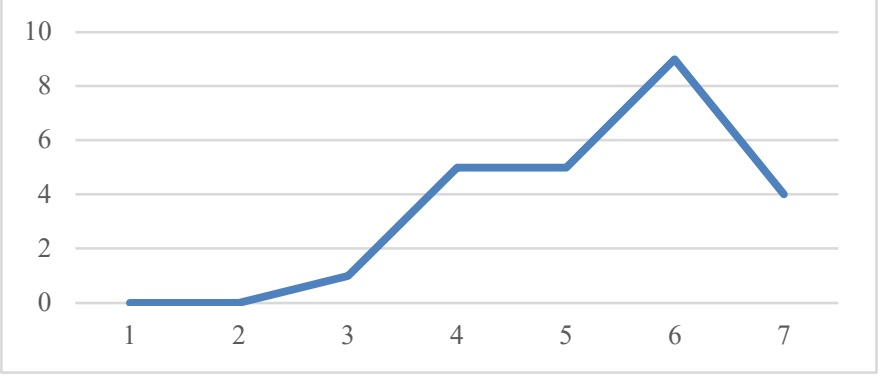

Figura 7. Pregunta 25 del cuestionario final.

Para cerrar esta sección, nos gustaría señalar que habiéndoles preguntado en el cuestionario final si estarían dispuestos a 
repetir la experiencia otro año de forma telemática con estudiantes de otra universidad, su respuesta fue mayoritariamente afirmativa. Como podemos ver en la figura 8 , la balanza se inclinó hacia los valores favorables en un 70,6\%, es decir, "bastante de acuerdo" (16,6\%), "muy de acuerdo" (25\%) y "totalmente de acuerdo" (29\%).

¿Estarías dispuesto a repetir esta experiencia y trabajar con estudiantes de otra universidad a distancia para crear un póster académico?

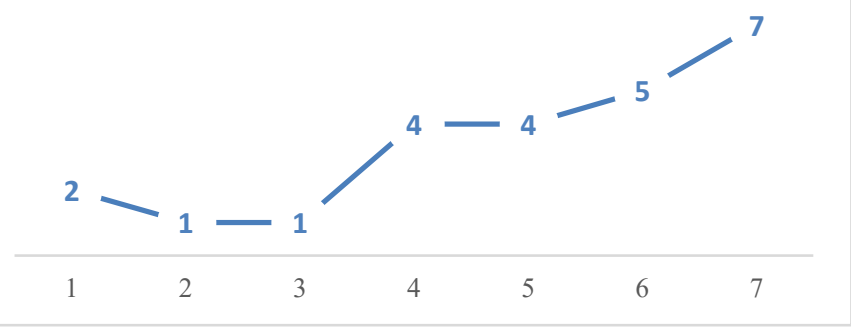

Figura 8. Pregunta 23 del cuestionario final.

De los resultados que aquí se presentan, se puede afirmar que el proyecto tuvo un impacto positivo dentro de la asignatura y que, pese a la inexperiencia de los estudiantes en la realización de un póster académico en inglés y su posterior presentación ante un público, el proyecto constituyó un factor motivador. Citando a uno de los estudiantes, había disfrutado "Because it is a different way to do it and it was more motivating" (Traducción de las autoras. "Porque fue una manera distinta de hacerlo y fue más motivador").

Además, quisiéramos señalar que, respecto al grado de consecución de las competencias transversales que se fomentan en la Universitat Politècnica de València, este proyecto de mejora docente tuvo un impacto considerable en varias de ellas. De los 27 estudiantes que contestaron este cuestionario específico, hubo tres competencias que destacaron sobre las demás. Estas se indican en la Tabla 1:

Tabla 1. Grado de adquisición de las competencias transversales.

\begin{tabular}{ll}
\hline Competencia transversal & $\begin{array}{l}\text { Porcentaje de } \\
\text { consecución }\end{array}$ \\
\hline $\begin{array}{l}\text { Diseño y proyecto. Diseñar, dirigir y evaluar } \\
\text { una idea de manera eficaz hasta concretarla en } \\
\text { un proyecto. }\end{array}$ & $77 \%$ \\
$\begin{array}{l}\text { Trabajo en equipo y liderazgo. Trabajar y } \\
\text { liderar equipos de forma efectiva para la } \\
\text { consecución de objetivos comunes, } \\
\text { contribuyendo al desarrollo personal y } \\
\text { profesional de los mismos. }\end{array}$ & $66,6 \%$ \\
$\begin{array}{l}\text { Comunicación efectiva. Comunicarse de } \\
\text { manera efectiva, tanto de forma oral como } \\
\text { escrita, utilizando adecuadamente los recursos } \\
\text { necesarios y adaptándose a las características de }\end{array}$ & $53 \%$ \\
la situación y de la audiencia. & \\
\hline
\end{tabular}

Por último, quisiéramos señalar que, respecto a las calificaciones obtenidas en esta parte de la asignatura, todos los estudiantes mejoraron su nivel de inglés, sobre todo en lo referente al conocimiento de vocabulario propio de la aeronáutica, tal y cómo se puso de manifiesto tras la realización de una prueba de nivel al inicio y al final de la asignatura.

\section{CONCLUSIONES}

La presentación de los trabajos y proyectos con el póster académico ofrece una visión panorámica de los distintos temas y contenidos presentados en la asignatura, así como un ambiente más distendido que promueve la interacción entre los participantes que se convocan en un lugar de reunión y de encuentro físico "real".

Podemos concluir, asimismo, que aprender a hacer frente a este género en las primeras etapas de la carrera universitaria es importante y debe tenerse en cuenta al diseñar cursos no sólo de ciencias sino también de lenguas para fines específicos. Como hemos podido comprobar con los datos presentados, este formato de aprendizaje aporta un valor añadido al contenido de la asignatura, prestándose a la adquisición de una serie de competencias transversales que, sin lugar a duda, serán de gran utilidad a los estudiantes en su vida profesional.

Estamos, por lo tanto, del todo de acuerdo con lo afirmado por Baird (1991, citado en D'Angelo, 2010) quien, como hemos mencionado en la introducción a este estudio, argumentó que la realización de pósteres académicos es adecuada para todo tipo de clases ya que promueven el aprendizaje colaborativo, fomentan la creatividad y el pensamiento independiente, y desarrollan habilidades de investigación y comunicación. Los datos obtenidos en nuestro estudio así lo reafirman.

Esta experiencia puede ser transferida a cualquier otra asignatura de cualquier titulación ya que, además de resultar innovadora y motivadora como hemos visto por los resultados obtenidos, ayuda a los estudiantes a desarrollar una serie de destrezas que les serán, con toda seguridad, útiles en su vida laboral. Cabe destacar el logro obtenido en lo referente a los objetivos marcados respecto a las competencias transversales ya que se fomentaron especialmente las que se perseguían con la experiencia.

Como colofón al proyecto, todos los pósteres académicos generados en este proyecto de innovación y mejora docente (en torno a 50) serán expuestos en el vestíbulo de la Escuela durante una quincena. Asimismo, los profesores implicados en el proyecto tienen intención de crear una base de datos con todos los pósteres que se vayan generando en las siete asignaturas que participan en el proyecto como iniciativa para la sostenibilidad de este

y fuente de inspiración para posteriores cursos.

\section{AGRADECIMIENTOS}

Este estudio forma parte de un Proyecto de Innovación para la Mejora Educativa que ha podido realizarse gracias a la financiación concedida por el Vicerrectorado de Estudios, Calidad y Acreditación de la UPV; a la formación del Instituto de Ciencias de la Educación y a la participación de los docentes de la ETSID, así como de su equipo directivo, muy especialmente, a Juan Antonio Monsoriu y a Vanesa Cuenca Gotor. 


\section{REFERENCIAS}

D'Angelo, L. (2010). Creating a framework for the analysis of academic posters. Language Studies Working Papers, 2, $38-50$

Billet, D. (2001). Ready for Take-Off: The Language of Aeronautics. Cannes: Media Training

García Folgado, María José (2017). El pòster com a gènere discursiu. Revista de didáctica de la llengua $i$ de la literatura, 73, 34-37.

García-Manso, A. (2019). El póster de biblioteca: hacia una resignificación del póster académico. MULTIárea. Revista de didáctica, (10), 101-113.

Hyland, K. (2000). Disciplinary Discourses: Social Interactions in Academic Writing. Harlow: Longman.

Halligan, P. (2008). Poster presentations: Valuing all forms of evidence. Nurse Education in Practice, 8(1), 41-45. https://doi.org/10.1016/j.nepr.2007.02.005

Moore, L. W., Augspurger, P., King, M. O., y Proffitt, C. (2001). Insights on the poster preparation and presentation process. Applied nursing research:
ANR, 14(2), 100-104. https://doi.org/10.1053/ap nr. 2001.22376

Navarro, S. M., y Félix, E. G. (2017). El uso del póster como técnica pedagógica. En Enseñanza centrada en el aprendizaje y diseño por competencias en la Universidad: fundamentación, procedimientos y evidencias de aplicación e investigación (pp. 265-284). Valencia: Tirant lo Blanch.

Rosell Clari, V., Trenado Santarén, R., y Simó Teufel, S. (2011). El poster como instrumento de evaluación en la adquisición de competencias específicas y transversales en el segundo curso del Grado de Psicología. Departamento de Psicología Básica. Universitat de València. https://pdfs. sem anticscholar .org/7a9c/01b 145932dc80e9f3951b58ce2ae994345c8.pdf

Schoepp, K., y Erogul, M. (2001). Turkish EFL Students' Utilization of Information Technology Outside of the Classroom. TEFL WebJournal, 1(1), 14.

Shelledy, D. C. (2004). How to make an effective poster. Respiratory Care, 49(10), 1213-1216. https://doi.org/10.15694/mep.2015.004.0001 